\title{
RESPONSABILIDAD SOCIAL DE LA UNNE Primero las necesidades comunitarias
}

\author{
MARTHA ELIZABETH ANTOLA \\ FACULTAD DE CIENCIAS ECONÓMICAS - UNNE
}

PRIMER PREMIO. Categoria: No Docentes

"La Educación es un medio, entre otros, pero más que otros, al servicio de un desarrollo humano más auténtico y más armonioso y, por tanto que haga retroceder la pobreza, la marginación, la ignorancia, la opresión y la guerra". (Informe para la UNESCO de la Comisión Internacional sobre Educación para el siglo XXI, 1996) 


\section{RESUMEN}

$\mathrm{n}$ el caso de la universidad pública argentina, la responsabilidad social es uno de
los ejes insoslayables de la Reforma Universitaria de 1918 cuyos valores se hacen
necesarios ampliar y actualizar respecto de las exigencias de nuestro tiempo,
particularmente teniendo en cuenta que la desigualdad social y la pobreza de nuestra región así lo exigen.

En esta monografía se presentará un resumen sobre la visión que tiene el Personal No Docente de la Universidad Nacional del Nordeste con relación a las prácticas en responsabilidad social y desarrollo humano, información que permitirá inferir el plan de acción formulado por las máximas autoridades de la universidad y los proyectos llevados a cabo por cada unidad académica.

Las fuentes de información seleccionadas reflejarán las distintas formas de representar el mismo problema (RSU), lo que implica diferentes posturas frente al mismo tema; estas representaciones permiten generar nociones de sentido, herramienta útil en la instancia del diseño de estrategias para enfrentar el relevante problema que se pretende analizar. En tanto, la metodología utilizada tiene sustento en el diagnóstico participativo.

En homenaje al $60^{\circ}$ Aniversario de la Facultad de Ciencias Económicas de la Universidad Nacional del Nordeste.

\section{INTRODUCCION}

El presente trabajo de investigación fue elaborado con la intención de profundizar en el estudio de la responsabilidad social universitaria, el cual ha surgido como nuevo desafío ante las actuales exigencias del contexto local, nacional e internacional. En esta búsqueda la universidad pública cuenta con excelentes oportunidades para lograr una integración verdadera entre los actores sociales y las experiencias solidarias universitarias propias y de otras instituciones.

La crisis de valores y el desinterés por el prójimo deterioran la calidad de vida y, en consecuencia, algunos actores sociales quedan fuera del sistema educativo y del mercado de trabajo, perdiendo con ello la dignidad humana.

Compartiendo plenamente lo expuesto por Berenblum, se sostiene que "así como hemos naturalizado o tolerado la pobreza extrema y la corrupción, también aprendimos a ser indiferentes a los problemas de nuestros compatriotas (con la excusa de tener nuestros propios problemas o trabajar demasiado y no tener tiempo para ocuparnos de los demás); es hora de reflexionar seriamente sobre nuestras conductas y nuestra forma de pensar, como seres humanos, como institución educativa o como sociedad en pleno crecimiento".

La UNNE puede y debe incentivar el compromiso social de sus miembros, llámense estudiantes, docentes, no docentes, cuerpo directivo; con nuestra participación en proyectos 
sociales estaremos contribuyendo a conseguir mayor educación, más conocimientos, mejor generación de riquezas y más igualdad y equidad en la distribución de recursos.

"Resulta imprescindible la vinculación entre las diversas universidades y la sociedad, para que el pensamiento crítico que se genera puertas adentro, pueda socializarse y materializarse en acciones concretas que repercutan positivamente en la vida de todos los miembros de la sociedad. La búsqueda de una sociedad más justa, que mejore la calidad de vida de todos, debe ser un objetivo primordial de la UNNE y su función social por excelencia. (...) A través de prácticas solidarias los alumnos no sólo devuelven a la sociedad la posibilidad de estudiar que reciben de ella, sino que a su vez transitan una experiencia de aprendizaje orientada a abordar profesionalmente problemáticas sociales concretas" (ARIAS, G. 2009).

En tal sentido las actividades de Extensión orientadas a la RSU permitirán “identificar los problemas y demandas de la sociedad y su medio, coordinar las correspondientes acciones de transferencia de conocimientos, reorientando y recreando actividades de docencia e investigación a partir de la interacción con ese contexto, como así también la interacción creadora entre universidad y comunidad, mediante la cual el quehacer cultural se vincula con el fenómeno social a fin de producir las transformaciones necesarias para el logro de una mejor calidad de vida" (Encuentro Institucional en la UNNE 2004).

Por lo anteriormente expuesto cabe preguntarnos: ¿cuáles son las nuevas estrategias para enfrentar la complejidad actual de los problemas sociales?; ¿cómo podríamos implementarlas en las prácticas cotidianas de la UNNE?; ¿con qué recursos?; como personas y como institución, ¿de qué manera hemos asumido el compromiso de la RSU?

\section{MARCO NORMATIVO}

\subsection{NORMATIVAS/ORGANISMOS}

Constitución Nacional

\section{PUNTOS CENTRALES /ACCIONES}

Art. $\mathrm{N}^{\circ}$ 14: “Todos los habitantes de la Nación gozan de los siguientes derechos conforme a las leyes que reglamenten su ejercicio; a saber: de trabajar y ejercer toda industria lícita; de navegar y comerciar; de peticionar a las autoridades; de entrar, permanecer, transitar y salir del territorio argentino; de publicar sus ideas por la prensa sin censura previa; de usar y disponer de su propiedad; de asociarse con fines útiles; de profesar libremente su culto; de enseñar y aprender."

\subsection{NORMATIVAS / ORGANISMOS}

Ley Nacional de Educación Superior $\mathrm{N}^{\circ} 24521$

\section{PUNTOS CENTRALES /ACCIONES}

Art. $3^{\circ}$ : "La Educación Superior tiene por finalidad proporcionar formación científica, profesional, humanística y técnica en el más alto nivel, contribuir a la preservación de la cultura nacional, promover la generación y desarrollo del conocimiento en todas sus formas, y desarrollar 
las actitudes y valores que requiere la formación de personas responsables, con conciencia ética y solidaria, reflexivas, críticas, capaces de mejorar la calidad de vida, consolidar el respeto al medio ambiente, a las instituciones de la República y a la vigencia del orden democrático."

Art. $\mathrm{N}^{\circ}$ 43: "Establece como prioridad para ser acreditadas aquellas carreras que pudieran comprometer el interés público, poniendo en riesgo de modo directo la salud, la seguridad, los derechos, los bienes o la formación de los habitantes."

\subsection{NORMATIVAS / ORGANISMOS}

Estatuto de la UNNE

\section{PUNTOS CENTRALES /ACCIONES}

Art. $1^{\circ}$ - Titulo Preliminar: Naturaleza y Fines. (...) "tiene por fin primordial la generación y comunicación de conocimientos del más alto nivel, y la formación ética, cultural, técnica y profesional, contribuyendo al esclarecimiento de los problemas y necesidades de todo orden de la región, para beneficio del hombre y extendiendo su acción y servicios a la Comunidad a la que pertenece, en un clima de libertad,justicia, igualdad y solidaridad".

Art. $2^{\circ}$ : (...) "Procura formar integral e interdisciplinariamente a sus componentes $e$ infundir en ellos el espíritu crítico y a la vez el sentido de responsabilidad personal y social de reafirmación ético cívica y de sus convicciones democráticas y republicanas, dentro de un marco de pluralidad y respetuoso disenso".

Art. $110^{\circ}$ : "La Universidad, mediante la extensión universitaria, promueve su inserción en el medio y proyección a la comunidad; participa de la responsabilidad de la labor educativa y de mejoramiento cultural del pueblo (...)”.

Titulo Quinto (art. 104 al art. 112) De la función social de la Universidad: "Procura la formación integral y armónica de sus componentes, y realiza en el seno de la sociedad una labor organizada y permanente, para propender a la dignificación integral del hombre, a la formación de una conciencia democrática vigoros a y esclarecida y a la capacitación cultural y técnica de sus componentes y del pueblo de que forma parte, como órgano e instrumento de mejoramiento social (...)”.

\subsection{NORMATIVAS / ORGANISMOS}

Ministerio de Educación de la Nación - Secretaría de Políticas Universitarias

\section{PUNTOS CENTRALES /ACCIONES}

Programa Nacional de Voluntariado Universitario (2006): Carta compromiso, duración: 6 meses a 1 año, inversión por programa: \$22.000.

\subsection{NORMATIVAS / ORGANISMOS}

Propuesta de Gobierno y Gestión para el periodo 2010-2014: "Gestión para la Excelencia con Responsabilidad Social e Innovación”. UNNE 


\section{PUNTOS CENTRALES /ACCIONES}

- Programa de investigaciones en temas estratégicos prioritarios regionales: pretende generar el conocimiento en aquellos temas que sean importantes para el desarrollo regional, a través de la investigación se ofrece a la sociedad la posibilidad de superar los problemas que la aquejan. Este programa tiene como objetivo identificar temas fundamentales para la región a efectos de incentivar a los docentes investigadores a trabajar en ellos. Los temas tentativos son: estudio integral del agua, energía, producción agropecuaria, pobreza, desarrollo local, desarrollo sustentable.

- Programa de Responsabilidad Social: el propósito fundamental de la Universidad es la formación humana y profesional y la construcción de nuevos conocimientos y en razón de estas actividades, la Universidad cumple un rol social de mucha importancia en el desarrollo de las sociedades. El objetivo de este Programa es profundizar la relación de la Universidad con el medio social, fomentar en la comunidad universitaria la implementación de acciones tendientes a corregir los déficits sociales, favorecer la generación de proyectos de desarrollo de alto impacto en el medio, en particular de los sectores más desfavorecidos, profundizar el conocimiento sobre los problemas sociales que afectan a la comunidad regional, fomentar la conformación de grupos de investigación interdisciplinarios para el estudio de la problemática social, formar profesionales para desempeñarse como especialistas en políticas sociales en organizaciones gubernamentales y no gubernamentales con aptitudes y habilidades para trabajar con equipos pluridisciplinarios para el abordaje integral de los problemas sociales en sus diferentes aspectos y dimensiones.

\subsection{NORMATIVAS / ORGANISMOS}

I Conferencia Mundial sobre la Educación Superior: "La educación superior en el siglo XXI: Visión y Acción" (1998)

Conferencia Mundial de Educación Superior 2009: “Las Nuevas Dinámicas de la Educación Superior y de la Investigación para el Cambio Social y el Desarrollo" - UNESCO (Paris 2009)

2.7 NORMATIVAS / ORGANISMOS

Programa de la Naciones Unidas para el Desarrollo

PUNTOS CENTRALES /ACCIONES

Informes sobre Desarrollo Humano (DH)

\subsection{NORMATIVAS / ORGANISMOS}

Red Latinoamericana de Socioeconomía Solidaria

PUNTOS CENTRALES /ACCIONES

Programa de alfabetización económica 


\subsection{NORMATIVAS / ORGANISMOS}

Maestría en Administración Pública: área de Gerencia Social (UBA-FCE)

\section{PUNTOS CENTRALES /ACCIONES}

Trabajos de investigación sobre proyectos sociales

\subsection{NORMATIVAS / ORGANISMOS}

Cátedra de Honor: Gerencia Social para el DH (UBA-FCE)

\section{PUNTOS CENTRALES /ACCIONES}

Trabajos de investigación sobre proyectos sociales

\section{3. ¿CÓMO ABORDA EL PROBLEMA DE LA RSU NUESTRA UNIVERSIDAD?}

Antes de analizar las prácticas de responsabilidad social de la UNNE corresponde introducir un nuevo elemento de análisis denominado Alteridad, "considerada como la medida de la conciencia, del registro que tenemos de los que nos rodean y de la importancia que les damos. El filósofo Tzvetan Todorov explicaba que la alteridad implica la capacidad de reconocer humanidad en los demás. El reconocimiento del otro como un ser humano válido, legítimo, que merece respeto y tiene los mismos derechos que uno, es la base de una convivencia pacífica y un requisito básico para el establecimiento del diálogo y el consenso. El ejercicio de la alteridad fomenta también la actitud solidaria hacia los más desprotegidos" (BEREMBLUN D.; 2009).

La responsabilidad social exige una toma de conciencia respecto del lugar que ocupan en la escala social, de aquellos que están llamados a asumirla por su desempeño en contextos organizacionales; ya sea el individuo dentro de la organización, como el lugar de la organización misma en sus distintos contextos. Nos referimos al espacio que ocupa, tanto en el marco de su actividad, en la cadena de valor en las que interviene, en los mercados donde participa; $y$ también del papel que juega en la interacción con su entorno físico, social, económico, político, etc.

Cuando este compromiso es asumido y se manifiesta en hechos concretos, esas acciones contribuyen a mejorar la calidad de vida promedio de la comunidad de regencia. El ejercicio de la RSU permite avanzar hacia comunidades más inclusivas e integradas, en una adecuación progresiva de las organizaciones para una mayor apertura a la complejidad de la dinámica social y sus necesidades emergentes.

Para poder ser socialmente responsables, es imprescindible conocer el contexto que nos rodea y comprender su historia (dónde estamos y cómo llegamos aquí). Porque el ejercicio de esta responsabilidad se vincula directamente con una autopercepción -situación en tiempo y espacio- del hombre como sujeto ético. Por eso, es fundamental que el individuo tome conciencia de su lugar en la sociedad y del alcance de sus decisiones, si quiere obtener una perspectiva realista" (SARABIA F.; 2009). Tanto el método del diagnóstico participativo como la fuente de información extraída de los foros de participación propuestos por esta cátedra “Organización y Administración de las Instituciones Universitarias -impartida por la Tecnica- 
tura en Administración y Gestión de Instituciones Universitarias de la UNNE- nos permitirán abordar el prolegómeno de la R.S.U. a partir de los siguientes interrogantes:

- Cuál es la situación problemática detectada?

- Cuáles son sus principales causas y consecuencias?

- Cómo se relaciona el problema con otros problemas?

- Qué piensa, siente, imagina la comunidad universitaria?

- Cómo el Personal No Docente de la UNNE percibe la idea de R.S.U.?

- Cuáles son las razones por las que se debería buscar una solución?

- Es la solución propuesta la más adecuada y viable?

A continuación se intentará responder principalmente la pregunta ¿̇cómo el Personal No Docente de la UNNE percibe la idea de responsabilidad social universitaria?

\section{UNIDADES ACADEMICAS ACCIONES}

\subsection{UNIDAD ACADEMICA}

UNNE

\section{ACCIONES}

En el Instituto Rectorado se encuentra la Secretaria de Asuntos Sociales, la cual es el área encargada de los requerimientos del estudiantado, desde ella la UNNE pretende trabajar sobre la idea de un nuevo contrato social. A diferencia del anterior éste demandará un mayor direccionamiento de las actividades de investigación vinculadas a las necesidades sociales.

La propuesta de gobierno y gestión de la Universidad es la "Gestión para la Excelencia con Responsabilidad Social e Innovación para el periodo 2010-2014”. Ello se afirma en su visión de futuro, "....la UNNE se define como una comunidad de trabajo, integrada por docentes, no docentes, estudiantes y graduados, anhelando constituirse en una institución totalmente abierta a las exigencias de su tiempo y de su medio en el más amplio contexto, con una imagen pública que se proyecte con respeto hacia la comunidad en virtud de los servicios que le ofrece y a su vez, sea respetada por ésta por su Responsabilidad Social ....”. También se encuentra planteado entre los principios y valores que deben guiar nuestro accionar.

Es así que se han:

- Firmado acuerdos de trabajo nacionales e internacionales: en los que se realizan actividades destinadas a la comunidad a favor de la lucha contra la pobreza, la protección del medio ambiente, la defensa de la vida democrática, el desarrollo global sostenible y equitativo en la región.

- Implementado aulas virtuales y extensiones áulicas de carreras en el interior del Chaco y de Corrientes.

- Las facultades de la UNNE a lo largo de sus años de vida fueron adaptando planes de estudios a los cambios lógicos de tecnologías y a los avances en los conocimientos científicos, asimismo han ido introduciendo asignaturas que ayudan al egresado a integrarse mejor al mundo laboral y social; algunas orientadas a entender la estructura que puede tener una orga- 
nización o los problemas humanos o de comunicación que pueden existir.

- UNNE Salud: se trata de pasantías realizadas por estudiantes de la carrera Bioquímica, junto a estudiantes de otras Facultades como Medicina, Odontología y Veterinaria, los que junto a profesionales y docentes, tienen la misión de contribuir al desarrollo saludable y sustentable de la región, en diversas localidades de Corrientes y de Chaco, previniendo enfermedades como: Dengue, Chagas, parasitosis, gripe A, anemia, lactancia materna, anticoncepción. Control de diversas enfermedades en zonas de bajos recursos y lugares alejados de los centros poblados, la tarea de este programa consiste en la vacunación de animales, vacunación de personas adultos y niños, asistencia y control bucal, análisis de diversas parasitosis tanto en personas como animales, control nutricional niños/adultos. Actividad realizada en coordinación con los Ministerios de Salud Pública de Chaco y Corrientes. Financiamiento del Ministerio de Salud de la Nación. Sitio web: http://www.unne.edu.ar/extension/unne_salud.php

- Las Puertas Abiertas: consiste en un programa de tipo educativo y cultural, que tiene la misión de extender los conocimientos, prácticas y actividades que la UNNE genera a favor del desarrollo social, cultural y económico de la región, mostrando el trabajo de investigadores, docentes, no docentes y alumnos, y fortaleciendo los vínculos con instituciones públicas y privadas. Sitio web: http://wiww.unne.edu.ar/extension/puertas.php

Radio universitaria: FM Universidad Nacional del Nordeste.

- La Universidad en el Medio: se trata de un programa de extensión de la universidad, que justamente promueve el desarrollo de proyectos de extensión a llevarse a cabo por equipos interdisciplinarios formados por docentes y alumnos que han aprobado por lo menos la mitad de su correspondiente plan de estudios, además de no docentes de la UNNE, y personal de otros organismos provinciales, nacionales e internacionales, públicos o privados, que tienen la misión de mejorar la calidad de vida, optimizando el uso de los recursos. Desde la Secretaría General de Extensión Universitaria se anunció que son 80 los Proyectos que serán financiados con recursos de la UNNE, a partir del corriente año 2011. Sitio web: http://www.unne.edu.ar/ extension/unne_enelmedio.php

- Educación y Seguridad Vial: se trata de un grupo de Sub-Programas tales como: 1) Cursos de Capacitación en Seguridad y/ o Educación Vial; 2) Curso de Post-gradoen Accidentología Vial; 3) Jornadas, Seminarios y Congresos de actualización; y 4) Servicios a terceros; que tienen la misión de concientizar sobre el valor de la seguridad y educación vial en la sociedad, modificando sus hábitos incorrectos. Sitio web: http://www.unne.edu.ar/extension/educacion_vial.php

- Sistema de Becas de Transporte (interurbano y urbano), de Prestación Efectiva de Servicios, Comedor y de Finalización de Estudios: permiten solucionar las situaciones de emergencia que atraviesan los estudiantes. Sitio web: http://www.unne.edu.ar/estudiantes/becas.php

- Ingreso para mayores de 25 años sin título secundario: a partir de 1996, excepcionalmente, los postulantes a ingresar a la UNNE, mayores de 25 años y sin título secundario, pueden inscribirse para una evaluación de sus antecedentes, a efectos de determinar su nivel de formación, y de superar dicha evaluación, adquieren la condición de aspirantes, para luego pasar una 
prueba que verificará si poseen las aptitudes y conocimientos necesarios para cursar satisfactoriamente la carrera elegida. Sitio web: http://wwiv.unne.edu.ar/estudiantes/ingresantes.php

- S.A.S.E.: se trata de un Sistema de Cobertura Médico Asistencial para los estudiantes de la UNNE que acrediten su condición con certificado de alumno regular, y para los respectivos cónyuges e hijos, abonando una determinada cuota. Sitio web: http://wwwv.unne.edu.ar/ estudiantes/obra_social.php

- Asistencia Social: el Departamento Universitario de Salud Estudiantil, de la Dirección General de Asuntos Estudiantiles, dependiente de la Secretaría General de Asuntos Sociales de la UNNE, ofrece servicios que tienen como finalidad, velar por la salud física, psíquica y espiritual del estudiante, con el propósito de contribuir al máximo rendimiento en sus actividades académicas. Los servicios referidos son: atención médica, odontológica, kinesiológica y bioquímica, obstetricia, actividades de prevención y promoción de salud, se dictan charlas y se elabora material ilustrativo sobre los distintos temas. Sitio web: http://www.unne.edu.ar/estudiantes/asistencia_social.php

- Programa UNNE Municipios: financiado con recursos de la Universidad.

- Programa de Pueblos Indígenas que plantea la inclusión de los mismos en carreras de grado y de profesorado, la capacitación en esta temática, el diseño de proyectos de extensión en función de la demanda de las comunidades y de proyectos de investigación sobre pueblos indígenas.

- Centro Cultural Nordeste y Centro Cultural Universitario: Teatro Universitario.

Proyecto Universidad del Sol - orientación vocacional: a través de este programa junto a un equipos de docente y psicólogos que van a diferentes escuelas del interior de la provincia y difunden las ofertas académicas, becas de estudios y alojamientos para estudiantes que deseen ingresar a la UNNE.

- Proyectos de investigaciones científicas, tecnológicas y humanísticas: se busca el bien social y la creación de conciencia ciudadana.

- Convenios de trabajo con entidades municipales, provinciales, nacionales, entes internacionales (Universidades de España).

- Sistema de Seguridad e Higiene en el Trabajo.

- Programas Estratégicos para el Desarrollo Sustentable.

- Proyectos de Voluntariado: con financiamiento de la Secretaría de Políticas Universitarias.

- Agencia de Innovación y Desarrollo para PYMES (AGENTIA): cuya misión es promover y gestionar conocimiento social y culturalmente pertinente en el campo interdisciplinario del desarrollo, para beneficio de la sociedad de la Región del Norte Grande.

- Dirección de Vinculación y Transferencia

- Centro de Estudios Sociales

- Centro de Gestión Ambiental y Ecología

- Incubadora de Empresas de base Tecnológica (UNNETEC-INNOVAR): destinada a apoyar el surgimiento y desarrollo de nuevas empresas que tengan un alto valor agregado de conocimiento e innovación.

Programas de accesibilidad para personas con discapacidades disminuidas: transfor- 
mar todos los edificios de la UNNE de manera que sea accesible para todos, a través de rampas de acceso, señalética con leguaje braile, planos apticos, señalética sonoras, mostradores de atención al público adaptados a las necesidades de estas personas. Los nuevos edificios que se construirán ya cuentan entre sus requisitos todas las pautas de accesibilidad.

- Cursos de Capacitación (Secretaria General de Extensión Universitaria).

\subsection{UNIDAD ACADEMICA}

HUMANIDADES

\section{ACCIONES}

- Extensión áulica en General Pinedo de la carrera del Profesorado en Educación Inicial, en Formosa con el Profesorado Universitario, diferentes posgrados.

Carreras de articulación: para egresados de nivel terciario de bibliotecarios y archivistas, para que puedan obtener su licenciatura universitaria, profesorados en Ciencias Jurídicas y Económicas para profesionales de las dos áreas, dando respuesta a muchos de estos profesionales que querían contar con el título habilitante para desempeñar la docencia.

- Ciclos de Perfeccionamiento para Profesionales: Ej. Profesorado en Ciencias Económicas y Profesorado en Ciencias Jurídicas para completar la formación docente de egresados de la UNNE en estas líneas de conocimiento.

- Programa de Pueblos Indígenas.

- Jornada de la Madres Cuidadoras Qóm: de la comunidad Toba.

- Curso "Aportes y Saberes de la Cultura Qóm": donde participaron como expositoras más de 12 Madres Qom, de la Localidad de Pampa del Indio - Chaco.

- Escuela Abierta

Convenios de capacitación, asistencia técnica. perfeccionamiento: con Instituciones del nivel medio y superior, públicas y privadas, tales como los Municipios de Chaco y Corrientes, a las que se ha asistido en el desarrollo de los SIG (Sistemas de Información Geográfica) para el planeamiento urbano y control catastral a través de seguimiento satelital.

El Instituto de Investigaciones en Educación ha trabajado o está trabajando en los siguientes proyectos:

- Percepciones y valoraciones de estudiantes y profesores en torno a la evaluación en un Instituto de Formación Docente.

- Patrones de organización de la información en las prácticas inferenciales.

- Desgranamiento temprano en la Facultad Regional Resistencia de la Universidad Tecnológica Nacional.

- Formación docente inicial y primeras experiencias laborales en escuelas de nivel medio.

- Perfiles y estilos de aprendizaje en estudiantes universitarios.

- Teorías ingenuas sobre la evolución biológica en la escuela. Sus convergencias y divergencias en relación a concepciones científicas.

- Dispositivos de acompañamiento en la formación docente. 
El acompañamiento y sus configuraciones en el ciclo de prácticas de la futura profesión.

Historia de la Universidad Popular de Resistencia. 1929 - 2005.

Historia de la Educación Bilingüe Intercultural en la Provincia del Chaco. 1984- 2006.

Proyecto RELEE - Redes de Estudios en Lectura y Escritura.

- Escuela media y trabajo docente en la provincia del Chaco. Dimensiones políticas, institucionales, culturales y sociales en la configuración del trabajo de los profesores y de las instituciones educativas.

La práctica de la investigación en las propuestas de enseñanza de la Metodología de la Investigación de las carreras del campo de Ciencias Sociales de la Universidad Nacional del Nordeste

El malestar docente y su relación con los procesos de éxito-fracaso escolar. Un estudio en el nivel medio de enseñanza.

Subjetividades urbanas violentas en el espacio público del Gran Resistencia.

Cabe destacar que las actividades de extensión hacia el medio se llevan a cabo enmarcadasen la base de una real calidad educativa, cumpliéndose para ello con las tres dimensiones que la refieren, y que se vinculan con los fines perseguidos por la UNNE.

\subsection{UNIDAD ACADEMICA}

ARQUITECTURA

\section{ACCIONES}

- Asesoramiento sobre proyectos de arquitectura al gobierno provincial o municipal.

- Estudios necesarios para la construcción de las viviendas populares o mejoramiento de las existentes.

- Aportes de los resultados de investigaciones sobre urbanismo.

- Campañas de prevención de enfermedades: tanto humanas como animales.

- Campañas de vacunación.

- Convenios de Capacitación. Asistencia Técnica. Perfeccionamiento: con Instituciones del nivel medio y superior, públicas y privadas, tales como Municipios de Chaco y Corrientes; a los cuales se los ha asistido en el desarrollo de los SIG (Sistemas de Información Geográfica) para el planeamiento urbano y control catastral a través de seguimiento satelital, siendo este un claro ejemplo de inserción en lo social y cotidiano de nuestra región.

- Restauración de obras de arte:

El Mural de la Reforma Universitaria que se encuentra en la Av. Rodríguez Peña, entre las calles Arbo y Blanco y San Lorenzo.

Perro Fernando (Patrono de Resistencia) en el Fogón de los Arrieros realizado por dos Arquitectos.

- Apoyo para que se declare a las esculturas de Resistencia Patrimonio de la Humanidad por parte de la Organización para la Educación, la Ciencia y la Cultura (UNESCO), dependiente de la ONU. 


\subsection{UNIDAD ACADEMICA}

INGENIERIA

ACCIONES

- Proyectos de Investigación y Extensión, en el marco de la convocatoria del Programa Universidad en el Medio:

"El manejo de los problemas ambientales en el ámbito escolar"

"Cocinas solares: alternativa tecnológica simple transferida para uso social y preservación del medio ambiente"

Sistema de Acción Tutorial: acompañando y guiando a los alumnos ingresantes para la inserción y adaptación a la cultura universitaria.

- Convenios de trabajo con distintas entidades del medio: municipales, provinciales, nacionales, internacionales (Universidades de México).

- Actividades de extensión con las colonias aborígenes: llevando conocimiento y tecnología.

- Cuidado del medio a mbiente: ha incorporado en todas las asignaturas temas referentes al Impacto Ambiental.

- Con orgullo podemos decir que casi toda la infraestructura de la ciudad y la región lleva la impronta de la Facultad de Ingeniería.

- Ha contribuido en la construcción del Puente Chaco-Corrientes, con la participación de un grupo de ingenieros de esta Facultad; esta obra es el primer puente argentino sobre el Río Paraná, no sólo comunicó a estas dos provincias, sino que potencializó la economía y el desarrollo de la Mesopotamia.

\subsection{UNIDAD ACADEMICA}

MEDICINA Y DEMÁS UNIDADES ACADÉMICAS O INSTITUTOS AFINES

\section{ACCIONES}

- Proyectos de investigación, docencia y extensión: nacionales, internacionales, zonales (Mercosur). Ejemplo Investigaciones con Pantallas Solares, a cargo del Departamento de Física, con el objetivo de lograr una solución al ahorro de energías eléctricas.

- Médicos Comunitarios.

- Servicio Universitario de Kinesiología.

- Servicio Universitario de Enfermería.

- Programa Nacional de Vacunación: dirigido a la comunidad universitaria, para prevenir distintas gripes invernales, trabajo en conjunto con las Municipalidades.

Convenios de Trabajos: asesoramiento y verificación de calidad de productos con las empresas Aguas de Corrientes, Municipalidad de la ciudad de Corrientes.

- Análisis de muestras extraídas para la detección del dengue, de leishmaniasis, etc.

- Consultorio Externo de Chagas.

- Charlas informativas sobre normas de higiene y prevención de la enfermedad transmitida por el mosquito dengue. 
- Programas de prevención odontológica y entregas de equipos odontológicos a los centros de salud en los barrios carenciados de la ciudad.

- Apoyo escolar para estudiantes de colegios secundarios: los estudiantes universitarios o docentes brindan clase de apoyo en esta materia durante los meses de receso escolar.

- ISSUNNE propone programas asistenciales y educativos en relación con la salud, priorizando el bienestar de sus afiliados en costo y servicios de calidad.

- UNNE Salud: mediante un convenio con la Municipalidad se realizan trabajo de campo en distintos barrios de la ciudad vacunando e informando sobre normas de higiene.

- PAMI

- Servicios asistenciales en el área del Hospital de Clínicas.

- Centro Nacional de Parasitología.

- Becas de grado, pregrado.

- Nuevas carreras de Postgrado y Especializaciones.

- Laboratorio de Bioquímica: disponible para todos aquellos que requieran sus servicios (tanto alumnos, docentes y No Docentes, como público en general).

\subsection{UNIDAD ACADEMICA}

CIENCIAS VETERINARIAS

\section{ACCIONES}

- Participa en proyectos, acuerdos, convenios, congresos, becas, además diseña políticas de investigación, estimula la difusión de sus actividades institucionales y los resultados de las investigaciones científicas, reflejados en publicaciones, comunicaciones científicas, congresos.

- Servicios de salud: clínicas, de salud pública, de producción, de sanidad de pequeños animales, de servicio asistencial de reproducción; poniendo a disposición los conocimientos, descubrimientos, equipamientos y recursos humanos a fin de cubrir todas las necesidades de la sociedad.

- UNNE- Salud: incluye programas de vacunación anti-rábica, control de leishmaniasis, etc.

- INICNE: control en especies ícticas.

- Hospital de Clínicas de Grandes: atiende especialmente a los equinos de los distintos ciudadanos que trabajan humildemente con sus carretas.

- Servicios de análisis de agua, de carne, bacteriológico, histopatológico.

- Educación sanitaria en barrios marginales.

- Unidad Ejecutora de Transferencia y Gestión Estratégica para el Desarrollo Sustentable.

- Unidad de Vinculación Tecnológica.

- Becas de investigación dirigida a "Temas Transferibles".

- Programa Universidad en el Medio.

- Programa UNNE-Municipios.

- Programa Nacional de Voluntariado Universitario (MECyT-SPU): cuyo propósito fundamental es de profundizar la vinculación de las Universidades Públicas e Institutos Universitarios Nacionales con la comunidad y, a su vez, incentivar el compromiso social de los estu- 
diantes de nivel superior universitario, promoviendo su participación voluntaria en proyectos sociales orientados a mejorar la calidad de vida de su población y estimular el desarrollo local.

\subsection{UNIDAD ACADEMICA}

CIENCIAS EXACTAS

\section{ACCIONES}

- Campaña de vacunación

- Campaña de fumigación en medio urbanos

\subsection{UNIDAD ACADEMICA}

DERECHO, CIENCIAS SOCIALES Y POLITICAS

\section{ACCIONES}

- Proyectos de investigación interdisciplinarios: se manifiesta en numerosas publicaciones locales, nacionales y extranjeras.

- Proyecto elaborado por docentes: Ej. El Dr. Víctor Hugo Benítez participó en la Cámara de Senadores de la Nación, en una reunión de especialistas, para debatir cambios en la caracterización de los delitos viales; propuso diversos proyectos que pretenden la inclusión como delitos viales de infracciones de tránsito que actualmente son contravenciones. La reunión tuvo como objetivo debatir sobre cómo agravar la pena de algunos delitos viales, y en particular se discutió respecto a cuatro proyectos de leyes ya presentados y que se pretende puedan avanzar en su curso parlamentario.

(http://corriente21.com.ar/profesional-de-la-unne-diserto-en-el-congreso-de-lanacion-sobreseguridad- vial/ - Publicado el Jueves, abril 14, 2011)

\subsection{UNIDAD ACADEMICA}

CIENCIAS ECONOMICAS

ACCIONES

Según expresiones vertidas por la Dirección de Gestión del Consejo Directivo de la Facultad de Ciencias Económicas, las acciones que contemplan la R.S.U. son:

“Acuerdo Complementario de Trabajo con la Facultad de Ciencias Económicas de la UBA para la implementación del "Programa Nacional de Formación en Ética para el Desarrollo. Premio AMARTYA SEN -PAS”. Tiene por finalidad central la formación de una nueva generación de profesionales a los más altos niveles de excelencia en nuevas áreas del conocimiento sobre el desarrollo y la ciencias gerenciales con perspectiva ética y del desarrollo humano y práctica de la inclusión social, multiplicando de esta forma a través de la tarea docente que desplieguen la introducción en las facultades de áreas de avanzada, claves para la formación de un profesional en ciencias económicas en el siglo XXI y en la Argentina de hoy.

- Se ha fijado como temas prioritarios de investigación, para la Convocatoria 2010 de las diferentes Becas de la Secretaria General de Ciencia y Técnica, los siguientes: 1) 
Distribución espacial de la pobreza: características, evolución y determinantes. Aplicaciones a los aglomerados urbanos regionales. 2) Determinantes y desempeño de políticas públicas en el ámbito regional. 3) Sistemas de información y evaluación económica de la Región NEA. Elementos y aplicaciones en desarrollo local.4) Geomarketing aplicado al desarrollo comercial de centros urbanos. 5) Impactos económicos y sociales de conglomerados competitivos. Desarrollo local. 6) Organización administrativa y de procedimientos. Adecuación de sistemas informáticos (UNNE-FCE). 7) Derecho del Consumidor. 8) Responsabilidad Social. 9) Análisis económico del Derecho. 10) Contabilidad y Auditoría de los impactos ambientales. 11) Los órganos de control del Estado. Su creación, características, clasificación y funciones. 12) Coparticipación. Aspectos políticos y económicos del federalismo fiscal.

- Se creó en el año 2010 la Secretaria de Investigación, Innovación y Desarrollo; entre sus objetivos principales se destaca el referido a acrecentar las relaciones Facultad-Sociedad, a través de la implementación de investigaciones científicas y tecnológicas pertinentes y su transferencia al medio.

- Se propuso al Consejo Superior de la Universidad la creación del Instituto de Investigaciones de la Sociedad, Instituciones y Empresa: organismo de investigación, docencia de posgrado y extensión, que se constituye para contribuir al análisis, esclarecimiento y propuesta de soluciones de los problemas científicos, académicos y técnicos de interés actual, con especial consideración de las necesidades económicas y sociales de la región".

Otros proyectos sociales son:

- Sistema de Pasantías

- Programa de becas

- Ayudas económicas para docentes: cursos y carreras de Postgrado

- Ayudas económicas para alumnos de grado: cursos

FUENTE: Foro de discusión $N^{\circ} 4$ "Responsabilidad social universitaria" Cátedra: Organización y Administración de las Instituciones Universitarias. Carrera: Tecnicatura en Administración y Gestión de Instituciones Universitarias - UNNE

\section{CONCLUSIONES}

Del análisis expuesto se concluye que la UNNE con su accionar aborda con cierto interés la R.S.U. y que ha incorporadola idea de GERENCIA SOCIAL en su agenda de gobierno; basada en experiencias sociales innovadoras busca contribuir al desarrollo humano, fundamentalmente se basa en la sustentabilidad de los proyectos, programas y procesos implementados.

Es oportuno destacar que para François Vallaeys, Profesor de Ética y Responsabilidad Social en CENTRUM-CATÓLICA, son cuatro los tipos de impactos universitarios: 


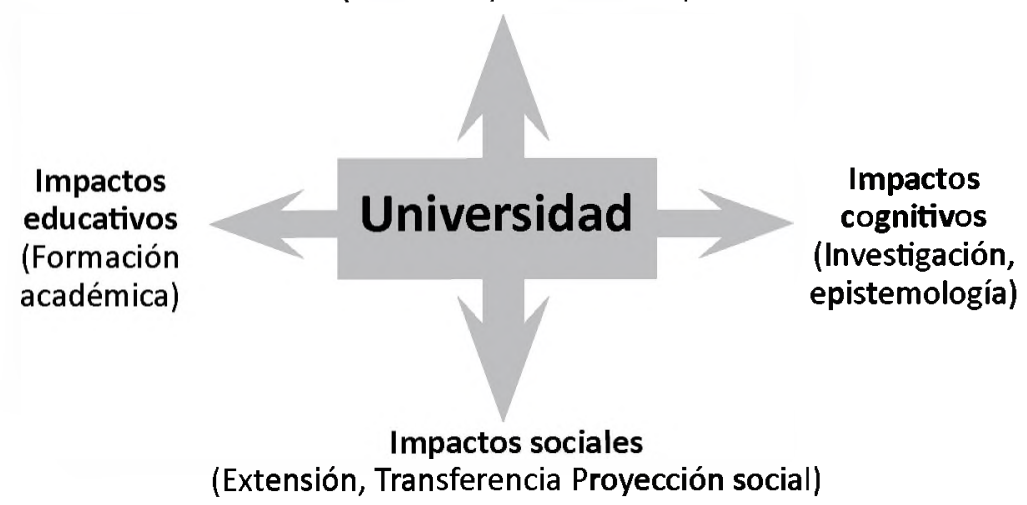

En la consecución de este loable objetivo la institución (UNNE) se encuentra ejecutando diversos programas sociales, para lo cual no se debería dejar de considerar las nuevas competencias actitudinales propuestas por Bernardo Kliksberg:

- Escuchar al otro como un legítimo otro, como un socio necesario, no como un adversario a ser neutralizado.

- Consensuar planes y acciones entre los participantes, con criterios de viabilidad en el corto y mediano plazo.

- Implementar las acciones convenidas, aunque no estén dadas las condiciones óptimas.

- Monitorear colectivamente y seguir los procesos hasta el logro de los resultados.

- Nunca olvidar agradecer la colaboración de todos, reconocer lo que no se pudo lograr y celebrar los resultados alcanzados.

En particular se destaca que la Facultad de Ciencias Económicas se encuentra transitando los primeros pasos hacia una Responsabilidad Social Universitaria considerada como política de mejor a continua dela institución, diseñada con miras al cumplimientoefectivo de su misión social y con fundamento en el concepto de alteridad (medida de la conciencia, del registro que tenemos de los que nos rodean y de la importancia que les damos; capacidad de reconocer humanidad en los demás; reconocimiento del otro como un ser humano válido, legítimo, que merece respeto y tiene los mismos derechos que uno; actitud solidaria hacia los más desprotegidos).

Pues bien, en los $60^{\circ}$ años de vida de la Facultad de Ciencias Económicas nosotros (la comunidad educativa en su totalidad) podríamos ofrecerle el más noble de los obsequios...

Convertir cegueras voluntarias en responsabilidad social ante la educación, el trabajo. la justicia y la dignidad humana; nueva actitud enfatizada en la generación. mantenimiento e incremento de la calidad de vida de aquellos que verdaderamente lo necesitan. 


\section{REFERENCIAS BIBLIOGRÁFICAS}

Ander Egg, E. Cómo elaborar un proyecto. Guía para diseñar productos sociales. Buenos

Aires. Editorial Magisterio del Río de la Plata. (1996)

Astorga Alfredo - Bart van der Bijl Berenblum, Daniel. Manual de diagnóstico participativo. Buenos Aires. Editorial Hvmanitas (1984) Capital social:proyectos sociales, proyectosen común. En Seminario de integración y aplicación en proyectos sociales. Compilado por FedericoSaravia. $1^{\circ}$ Edic. Buenos Aires. UBA. (2009)

Covey, Stephen. Los tres papeles que desempeña el lider en el nuevo paradigma. (1996) En: HESSELBEIN El líder del futuro.

Drucker, Peter. El líder del futuro. Bilbao. Ediciones Deusto. (1996)

Drucker, Peter. La organización del futuro. Buenos Aires. Ediciones Granica. (1998)

Drucker, Peter. La comunidad del futuro. Barcelona. Ediciones Granica. (1999)

Gesell, Silvio. El orden económico natural: hacia la libre tierra y la libre moneda.

Hillman, James. Tipos de poder. Guía para pensar por uno mismo. Buenos Aires. Ediciones Granica. (2000)

Innerarity, D. Ética de la hospitalidad. Barcelona. Ediciones Península. (2001)

Kliksberg, Bernardo. Pobreza, un tema impostergable. Buenos Aires. FCE (1993)

Kliksberg, Bernardo. La lucha contra la pobreza en América Latina. Buenos Aires. FCE (2000)

Kliksberg, Bernardo. La agenda ética pendiente de América Latina. Buenos Aires. FCE (2005)

Kliksberg, Bernardo. Capital social y cultura. Buenos Aires. FCE (2000)

Kliksberg, Bernardo. Pensamiento social estratégico. Buenos Aires. Siglo XXI. (2008)

Kliksberg, Bernardo. Primero la gente. Barcelona. Deusto. (2009) 
Lee, Blaine. El principio del poder. Cómo influir en los demás con honor. Barcelona. Grijolbo-Mondadori (2000)

Nirenberg, O - Brawerman J. - Programación y evaluación de proyectos sociales. Buenos Aires. Editorial Praidós. (2006).

Salomon, Robert. Nuevas reflexiones acerca de las organizaciones de negocios. El éxito basado en la integridad de las personas. México. Oxford University Press. (1999)

Sarabia, Federico. Seminario de integración y aplicación en proyectos sociales. Compilado por Federico Sarabia. $1^{\circ}$ Ed. UBA. Buenos Aires (2009)

Shaw de Critto, s. - Karl I. Voluntariado, una forma de hacer y de ser. Buenos Aires. Ediciones CICCUS. (1998)

Toledo, Ricardo Luis. Argentina sociedad anónima. (2007)

\section{CURRÍCULUM VITAE}

Contadora Pública Nacional. Profesora en Ciencias Económicas. Jefe del Departamento Tesorería - Facultad de Ciencias Económicas de la UNNE. Alumna de la Maestría en Ciencia Política en la Facultad de Derecho y Ciencias Sociales y Políticas, UNNE, con tesis en evaluación. Alumna de la Especialización en Tributación en la Facultad de Ciencias Económicas UNNE. Alumna de la Tecnicatura en Administración y Gestión de Instituciones Universitarias, en la Facultad de Ciencias Económicas, UNNE.

marthaantola@yahoo.com 